\title{
Manganese-enhanced MRI enables longitudinal tracking of transplanted stem cell viability in the murine myocardium
}

\author{
Rajesh Dash ${ }^{1,3^{*}}$, Aditya Subramanian ${ }^{1}$, Yuka Matsuura' ${ }^{1}$, I Suk Sohn ${ }^{1}$, Ting-Yuan Yeh ${ }^{1}$, Michael V McConnell ${ }^{1,2}$, \\ Joseph $\mathrm{Wu}^{1,3}$, Phillip Yang ${ }^{1,3}$
}

From 17th Annual SCMR Scientific Sessions

New Orleans, LA, USA. 16-19 January 2014

\section{Background}

Stem cell therapy in the heart is limited by an inability to track transplanted cell survival. To address this limitation, we used human amnion-derived mesenchymal stem cells (hAMSCs), which exhibit longer in vivo survival, and Manganese (Mn2+)-Enhanced MRI (MEMRI), which enters live stem cells to augment T1 signal. We tested $\mathrm{Mn} 2+$ pre-labeling of hAMSCs in vitro and whether MEMRI would detect hAMSC survival in mouse myocardium in vivo.

\section{Methods}

hAMSCs were isolated from human placentas after IRB consent. A subset of cells was transduced with a luciferase reporter gene. One group of hAMSCs was exposed to $1 \mu \mathrm{M}$ doxorubicin (DOX) for $4 \mathrm{hrs}$, then incubated for $48 \mathrm{hrs}$. The hAMSCs (Healthy \& DOX) were then labeled with increasing concentrations (0.1, 0.5 , \& $1 \mathrm{mM}$ ) of $\mathrm{MnCl} 2$ (Sigma, Inc) for $30 \mathrm{~min}$. Bioluminescence (BLI) was performed after $\mathrm{MnCl} 2$ labeling. Cells were pelleted into Eppendorf tubes and in vitro 3T MRI was performed (SignaHDx, GE, Inc). For in vivo MEMRI, $0.25 \times 106$ Healthy and DOX hAMSCs were pre-labeled with $0.5 \mathrm{mM} \mathrm{MnCl} 2$ for 30 min, washed, and pelleted for direct injection into hindlimb \& myocardium. Mice were immediately imaged using an FGRE-irP sequence: FOV4/ST $1 \mathrm{~mm} /$ $\mathrm{TE} \mathrm{min} / \mathrm{TI} 400 \mathrm{~ms} / \mathrm{NEX} 4$. In vivo MEMRI was repeated 2 days later, after $250 \mu \mathrm{l}$ of i.p. $\mathrm{MnCl} 2$.

\section{Results}

$0.5 \mathrm{mM} \mathrm{MnCl} 2$ increased the T1 signal and contrast-tonoise ratio (CNR) of healthy hAMSCs $\left(\triangle \mathrm{CNR} 80 \pm 2^{*}\right) \sim 3 \mathrm{x}$ vs DOX hAMSCs $\left(29 \pm 2,{ }^{*} \mathrm{p}<0.05\right)$ due to BLI-verified cell dropout. BLI showed no reduction in hAMSC survival after Mn2+ labeling (Figure 1A). Hindlimb imaging showed increased MEMRI CNR $(18 \pm 3)$ and BLI signal from pre-labeled hAMSCs (1B). Cardiac MEMRI of Healthy hAMSCs showed positive signal immediately after delivery as well as 2 days later (1C). However, DOX hAMSCs showed no MEMRI signal in vivo.

\section{Conclusions}

MEMRI successfully labels and tracks live, transplanted hAMSCs in the heart, enabling serial tracking of cell delivery and survival with no genetic pre-modification.

\section{Funding}

NIH K08 (RD) NIH R01 (PY) Stanford CVI Seed Grant (RD). 


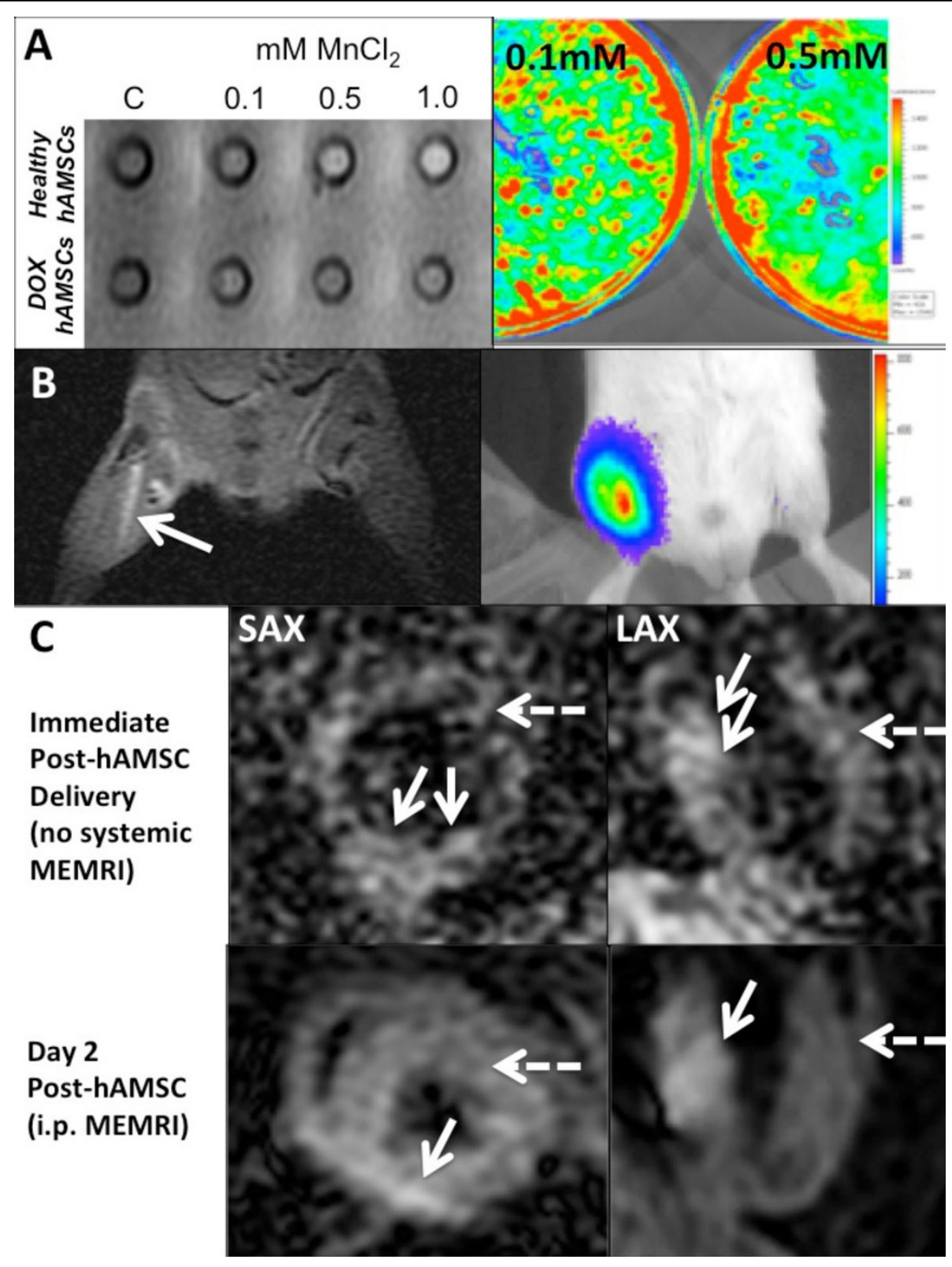

Figure $1 \mathrm{~A}$ : (left): $0.5 \mathrm{mM} \mathrm{MnCl} 2$ was sufficient to increase the T1 signal of pre-labeled healthy hAMSCs, but injured/dead DOX hAMSCs failed to take up MnCl2; (right) BLI shows better survival of hAMSCs 48 hrs after incubation with either $0.1 \mathrm{mM}$ or $0.5 \mathrm{mM}$ MnCl2, compared to DOX hAMSCs. 1B: (left) Hindlimb MRI showing positive MEMRI signal from pre-labeled hAMSCs in left hindlimb; (right) corresponding positive BLI signal from same hindlimb, confirming live hAMSCs. 1C: (top) short- (SAX) and long- (LAX) axis MEMRI of mouse heart immediately post-hAMSC delivery. Note the positive signal in the basal inferior wall (arrow) whereas simultaneous DOX hAMSC injection in anterior wall shows no MEMRI signal (dotted arrow); (bottom) 2 days after hAMSC delivery, i.p. MEMRI injection reveals intense uptake in basal inferior wall (healthy hAMSCs, arrow) with no uptake in anterior wall (DOX hAMSCs, dotted arrow).

\section{Authors' details}

${ }^{1}$ Cardiovascular Medicine, Stanford University, Stanford, California, USA. ${ }^{2}$ Engineering, Stanford University, Stanford, California, USA. ${ }^{3}$ Cardiovascular Institute, Stanford University Medical Center, Stanford, California, USA.

Published: 16 January 2014
doi:10.1186/1532-429X-16-S1-095

Cite this article as: Dash et al:: Manganese-enhanced MRI enables longitudinal tracking of transplanted stem cell viability in the murine myocardium. Journal of Cardiovascular Magnetic Resonance 201416 (Suppl 1):095. 